\title{
Long Term Results of High Dose Rate Brachytherapy and External Beam Radiotherapy for Local and Locally Advanced Prostate Cancer
}

\author{
Antonio Cassio Assis Pellizzon*, Deivid Augusto da Silva, Ricardo Cesar Fogaroli, Maria Leticia Gobo Silva, Douglas de Castro \\ Guedes, Guilherme Rocha Melo Gondim and Michael Chen
}

*Radiation Oncology Department, AC Camargo Cancer Center, Sao Paulo, Brazil

${ }^{\star}$ Correspondence to: Antonio Cassio Assis Pellizzon, Radiation Oncology Department, AC Camargo Cancer Center, Sao Paulo, Brazil, Tel: +55-11-21865105;

E-mail: acapellizzon@accamargo.org.br

Received: December 12, 2016; Accepted: January 02, 2017; Published: January 31, 2017;

\begin{abstract}
Purpose: Several studies provided evidence for the efficacy of dose-escalation on biochemical control (BC) of prostate cancer and High-dose-rate brachytherapy (HDR) is one method for it.

Materials and Methods: Patients with histological diagnosis Gleason scored (GS), clinical stage T1 to T3a, no evidence of metastatic disease, prostate volume $<60 \mathrm{cc}$ and initial PSA (PSAi) $<60 \mathrm{mg} / \mathrm{ml}$ were eligible.

Results: From 1997 to 2005 there were 273 patients treated with this treatment combination at AC Camargo Cancer Center, Sao Paulo, Brazil. The median age and FU time were 64.7 and 10.3 years, respectively. Two hundred thirteen (78.0\%) patients had FU longer than 5 years. Actuarial 10-year overall survival (OS), Clinical Specific Survival (CSS) and BC were 89.8\%, 63.6\% and 71.8\%, respectively. On univariate analysis GS $<7$, clinical stage $<\mathrm{T} 2 \mathrm{~b}$, low risk group (LR), absence adjuvant androgen deprivation (ADT), age $>65$, PSAi<10, localized EBRT and 3D-HDR plan were associated with improved CSS and BC, excluding PSAi, age for the last one. Multivariate Cox regression analysis confirmed LR, GS $<7$, PSAi $<10$, absence of ADT, age $<65$ years as predictors of improved CSS and BC. For OS only LR was confirmed as predictive factor.
\end{abstract}

Conclusion: The present data represents a unique uni-institutional study at long FU for the given technique. A comparison with the current literature confirms the excellent results achieved with this treatment modality. HDR has also the advantage of treatment time reduction and increasing in the capability of work load of the linear accelerators, especially in developing countries, where waiting lists and lack of radiation oncology facilities are a reality.

Key words: prostate cancer, radiotherapy, high-dose rate brachythrerapy, biochemical control, PSA

\section{Introduction}

More than $62 \%$ of Prostate Cancers (PCa) are diagnosed in men over 65 years. It has become a public health and socioeconomic problem with increasing incidence, in special due to a rapidly aging population worldwide [1]. In Brazil it was expected the diagnosis of 61,200 new cases of PCa in 2016, and the crude mortality for 2013 was around 14,000 deaths [2].

Management options for localized and locally advanced PCa are controversial and include active surveillance, radical prostatectomy, external beam radiotherapy (EBRT) and brachytherapy with low or high dose rate sources. [3]

Several studies provided evidence for the efficacy of doseescalation on biochemical control (BC) of PCa. Mature results from randomized trials show a direct relation between increasing the radiation dose given to the prostate and/or seminal vesicles and $\mathrm{BC}$ [4-7].
High-dose-rate after loading brachytherapy (HDR) is one method that can deliver a high localized radiation dose to the tumor with excellent BC when combined to EBRT [8]. One prospective randomized trial with up to 10 years follow up has proved that HDR plus EBRT is more efficient than EBRT alone in terms of BC with less acute rectal toxicity and improved quality of life [9].

The aim of this retrospective study is to evaluate the mature results of patients with local and locally advanced PCa treated with combination of HDR and EBRT.

\section{Materials and Methods}

Patients with confirmed histological diagnosis Gleason scored (GS) of PCa, AJCC clinical stage T1 to T3a, with no evidence of metastatic disease and initial PSA $<60 \mathrm{mg} / \mathrm{ml}$, prostate volume $<60$ $\mathrm{cc}$ measured by transrectal ultrasound (TRUS) suitable for EBRT and HDR under spinal anesthesia were eligible. Prior to treatment, patients 
had baseline investigations including pelvic computed tomography (CT) and/or magnetic resonance imaging, isotope bone scan, chest $\mathrm{X}$-ray and serum PSA. Exclusion criteria were evidence of metastases, co-existing malignancy or medical condition that precluded spinal anesthesia.

This single-centre institutional protocol of treatment was performed in compliance with the Declaration of Helsinki and approved by the local research Ethics Committee. Written informed consent was mandatory.

\section{External Beam Radiotherapy}

The EBRT target volume was defined using diagnostics CT images on conventional two dimensional or 3D planning. The targets were the prostate gland and the proximal seminal vesicles with a 1 to $1.5 \mathrm{~cm}$ margin except to the posterior region, which margins were reduced to 0.5 to $1.0 \mathrm{~cm}$. The EBRT dose ranged from 45 to $54 \mathrm{~Gy}$ prescribed to the intersection point. Further details of the radiotherapy schedules have been published previously [10].

\section{High Dose Rate Brachytherapy}

HDR was done under spinal anesthesia. Using TRUS with a perineal template affixed to perineum the exact needles positions were determined intraoperatively. In a first moment treatments were planned based on semi-orthogonal X-rays - two dimensional planning (2D) - and after that we moved to three dimensional (3D) planning, based on CT images. The prostate gland, the rectum, and the urethral trajectory and length were countered and identified in both situations. Implant dosimetry geometric optimization was initially utilized, followed later by use of inverse planning. Treatment parameters and dose constraints changed minimally throughout the years. The patients considered low risk had 16 Gy given in 4 fractions BID, one single implant. Intermediate and high risk patients had 20 Gy given in the same treatment schedule. The dose-volume histogram constraints were as follows: the TRUS or CT-based prostate's volume receiving $100 \%$ of the dose (V100) should be $>95 \%$, the uniformity index should be more than $50 \%$, and the V150 less than $30 \%$. The urethra maximum punctual and the maximal dose to $1 \mathrm{cc}$ of anterior rectal wall should not exceed $135 \%$ and $75 \%$ of prescribed doses, respectively.

\section{Definition of end points and statistical analysis}

BC was measured using PSA tests and assessed according to the Phoenix definitions [11]. Clinical Specific Survival (CSS) was calculated from the start of treatment to the lost of $\mathrm{BC}$, diagnose of metastatic disease or death from PCa. The BC was evaluated from the date of start the treatment until date of first biochemical failure. The follow up (FU) program also included clinical investigation, digital rectal examination and image studies.

The statistical program SPSS (statistical package for the social sciences) Inc., released 2008, Statistics for Windows, version 20.0 (SPSS Inc., Chicago, IL) was used for all statistical analysis. The analysis of OS, CSS and BC was made using the Kaplan-Meier method. The logrank test was used to test the significance when comparing different subgroups. Univariate and multivariate Cox regression analysis were also performed. The alpha level considered for statistically significant differences was 0.05 .

\section{Results}

Between March, 1997 and March, 2005 there were 305 patients treated with combination of HDR and EBRT at the Department of Radiation Oncology, AC Camargo Cancer Center, Sao Paulo, Brazil. Thirty two patients were lost of FU and the data of 273 patients was available for analysis. Sixty four (27.1\%) patients had pelvic EBRT and the remaining 209 (76.5\%) localized EBRT. Clinical and treatments characteristics are depicted in Tables $\mathbf{1}$ and 2.

Table 1: Patients Characteristics

\begin{tabular}{|c|c|c|c|c|c|c|}
\hline & & Median & Range & Variable & n & $\%$ \\
\hline & Age (years) & 64.7 & $42-82$ & & & \\
\hline & Prostate Vol (cc) & 36.3 & $19-72$ & $<35$ & 121 & 44.3 \\
\hline & & & & $>35$ & 152 & 55.7 \\
\hline & PSAi (ng/ml) & 10.3 & $1-52$ & $<10$ & 173 & 16.8 \\
\hline & & & & $10-20$ & 54 & 19.8 \\
\hline & & & & $>20$ & 46 & 63.4 \\
\hline & Gleason Score & & & $<7$ & 190 & 69.6 \\
\hline & & & & $=7$ & 58 & 21.2 \\
\hline & & & & $>7$ & 25 & 21.2 \\
\hline & & & & Yes & 47 & 17.2 \\
\hline & & & & No & 226 & 82.8 \\
\hline & Clinical Stage & & & $<\mathrm{T} 2 \mathrm{~b}$ & 192 & 70.3 \\
\hline & & & & $\mathrm{T} 2 \mathrm{~b}-\mathrm{c}$ & 47 & 17.2 \\
\hline & & & & $>\mathrm{T} 2 \mathrm{c}$ & 34 & 12.5 \\
\hline & Risk Group & & & Low & 133 & 48.7 \\
\hline & & & & Interm & 76 & 27.8 \\
\hline & & & & High & 64 & 23.4 \\
\hline \multirow[t]{5}{*}{ ADT } & NAAD & & & Yes & 93 & 34.1 \\
\hline & & & & No & 180 & 65.9 \\
\hline & ADJ & & & & 91 & 33.3 \\
\hline & Salvage & & & & 37 & 13.6 \\
\hline & WO & & & & 145 & 53.2 \\
\hline \multirow[t]{6}{*}{ EBRT } & Pelvic & & & & 64 & 23.4 \\
\hline & Localized & & & & 209 & 76.6 \\
\hline & Comorbidities & & & No & 146 & 53.5 \\
\hline & & & & SAH & 42 & 15.4 \\
\hline & & & & Diabetes & 19 & 7.0 \\
\hline & & & & Other & 39 & 14.3 \\
\hline TOTAL & & & & & 273 & 100.0 \\
\hline
\end{tabular}

Legend: ADJ: (adjuvant hormonal therapy), ADT (Androgen deprivation therapy), BF (Bichemical failure, EBRT: (External beam radiotherapy), NAAD (neoadjuvant hormonal therapy), SAH (Systemic arterial hypertension), Salvage: (Salvage hormonal therapy)

Table 2: Treatment Characteristics

\begin{tabular}{|l|l|c|c|c|c|c|}
\hline & & Median & Range & Variable & $\mathbf{n}$ & $\mathbf{\%}$ \\
\hline Dose & EBRT & 50 & $40-54$ & $<50$ & 149 & 54.6 \\
\hline & & & & $>50$ & 124 & 45.4 \\
\hline & HDR & & & 16 & 133 & 48.7 \\
\hline & & 18.3 & \multirow{2}{*}{$16-20$} & 20 & 140 & 51.3 \\
\hline & HDR plan & & & $2 \mathrm{D}$ & 167 & 61.2 \\
\hline & & & & $3 \mathrm{D}$ & 106 & 38.8 \\
\hline & Interval & 18.5 & $9-61$ & $<18$ & 173 & 63.4 \\
\hline & & & & $>18$ & 100 & 36.6 \\
\hline
\end{tabular}

Legend: EBRT (External beam radiotherapy), HDR (High-dose-rate brachytherapy), HDR plan 2D/3D (two or three dimensional planning) 
The median age and FU time were 64.7 (range, 42-82) and 10.3 (range, 1-15) years, respectively. Two hundred thirteen (78.0\%) patients had FU longer than 5 years, and of these 153 (56.1\%) longer than 10 years.

\section{Androgen deprivation therapy}

Androgen deprivation therapy (ADT) in a short course neoadjuvant $\mathrm{ADT}$, was prescribed for less than 6 months. Neoadjuvant hormonal therapy (NAAD) was administered to $34.1 \%$ of the patients. Adjuvant hormonal therapy (ADJ) was observed, mostly, for intermediate and high risk patients (33.4\%), generally for no more than 6 months for intermediate risk and up to 3-years in high risk patients. Salvage ADT was observed in 37 (90.2\%) of 41 patients dead due PCa. The profile of hormonal therapy is shown in Tables 3 and 4.

Table 3: Neoadjuvant Hormonal therapy according to Risk Group - Risk NAAD Cross tabulation

\begin{tabular}{|l|c|c|c|c|c|c|}
\hline \multirow{2}{*}{ Risk Group } & \multicolumn{2}{|c|}{ WO \% } & \multicolumn{2}{c|}{ YES \% } & \multicolumn{2}{c|}{ Total \% } \\
\hline Low & 74 & 27.1 & 8 & 2.9 & 82 & 30.0 \\
\hline Interm & 65 & 23.8 & 35 & 12.8 & 100 & 36.6 \\
\hline High & 41 & 15.0 & 50 & 18.3 & 91 & 33.3 \\
\hline Total & 180 & 65.9 & 93 & 34.1 & 273 & 100 \\
\hline
\end{tabular}

Legend: ADJ (adjuvant hormonal therapy), Interm (intermediate), NAAD (neoadjuvant hormonal therapy), WO (without hormonal therapy).

Table 4: Adjuvant and Salvage Hormonal therapy according to Risk Group

\begin{tabular}{|l|c|c|c|c|c|c|c|c|}
\hline & \multicolumn{2}{|c|}{ WO \% } & \multicolumn{2}{c|}{ ADJ \% } & \multicolumn{2}{c|}{ Salv \% } & \multicolumn{2}{c|}{ Total \% } \\
\hline Low & 72 & 26.4 & 7 & 2.6 & 3 & 1.1 & 82 & 30.0 \\
\hline Interm & 57 & 20.9 & 34 & 12.5 & 9 & 3.3 & 100 & 36.6 \\
\hline High & 16 & 5.9 & 50 & 18.3 & 25 & 9.2 & 91 & 33.3 \\
\hline Total & 145 & 53.2 & 91 & 33.4 & 37 & 13.6 & 273 & 100 \\
\hline
\end{tabular}

Legend: ADJ (adjuvant hormonal therapy), Interm (intermediate), NAAD (neoadjuvant hormonal therapy), Salv (Salvage hormonal therapy), WO (without hormonal therapy).

The crude 10-year overall survival (OS) rate at was $52.7 \%$. Actuarial 5- and 10-year OS, CSS and BC were $80.1 \%, 89.8 \%, 83.7 \%$, $63.6 \%, 85.5 \%$ and $71.8 \%$, respectively. (Figures 1-3)

\section{Univariate and multivariate analysis}

On univariate analysis GS $<7$, clinical stage $<\mathrm{T} 2 \mathrm{~b}$, low risk group, absence adjuvant ADT, older age ( $>65$-years), PSAi $<10 \mathrm{ng} / \mathrm{ml}$, localized EBRT and 3D HDR plan were favorable predictors of CSS. For $\mathrm{BC}$ all above have also confirmed as favorable predictors of $\mathrm{BC}$, excluding PSAi, age and localized EBRT ( $\mathrm{p}=\mathrm{ns})$. Table 5.

Univariate analysis failed to identify neoadjuvant androgen deprivation therapy (NAAD) as a predictor for BC in all group risks $(\mathrm{p}=\mathrm{ns})$. When we pooled the intermediate and high risk group into a unique denominated unfavorable risk group, NAAD also failed to predict improved CSS and BC.

Multivariate Cox regression analysis confirmed low risk group (HR 0.03, 95\% CI 0.006-0.116, $\mathrm{p}<0.001$ ) and intermediate risk (HR $0.09,95 \%$ CI $0.042-0.216, \mathrm{p}<0.001)$ compared to high risk, presence of ADT (HR 0.39, 95\% CI 0.179-0.868, p=0.021) as favorable predictors for CSS. GS $>7$ (HR 3.24, 95\% CI 1.279-8.220, $\mathrm{p}<0.001$ ), PSA $>10$ (HR $6.19,95 \%$ CI 2.015-19.041, $\mathrm{p}=0.001$ ), age $>65$ years (HR 2.87, 95\% CI 1.264-6.504, $\mathrm{p}=0.012)$ and EBRT dose $>50 \mathrm{~Gy}$ (HR 14.50, 95\% CI $1.874-112.164, \mathrm{p}=0.010$ ) were confirmed as adverse predictors for CSS. Tables 6-8, Figures 4-9.

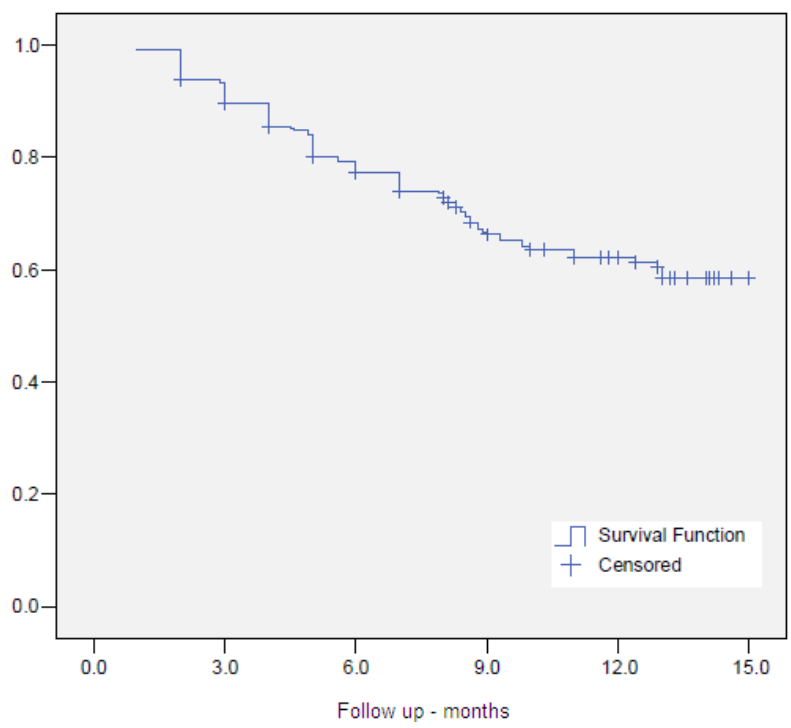

Figure 1: Overall Survival

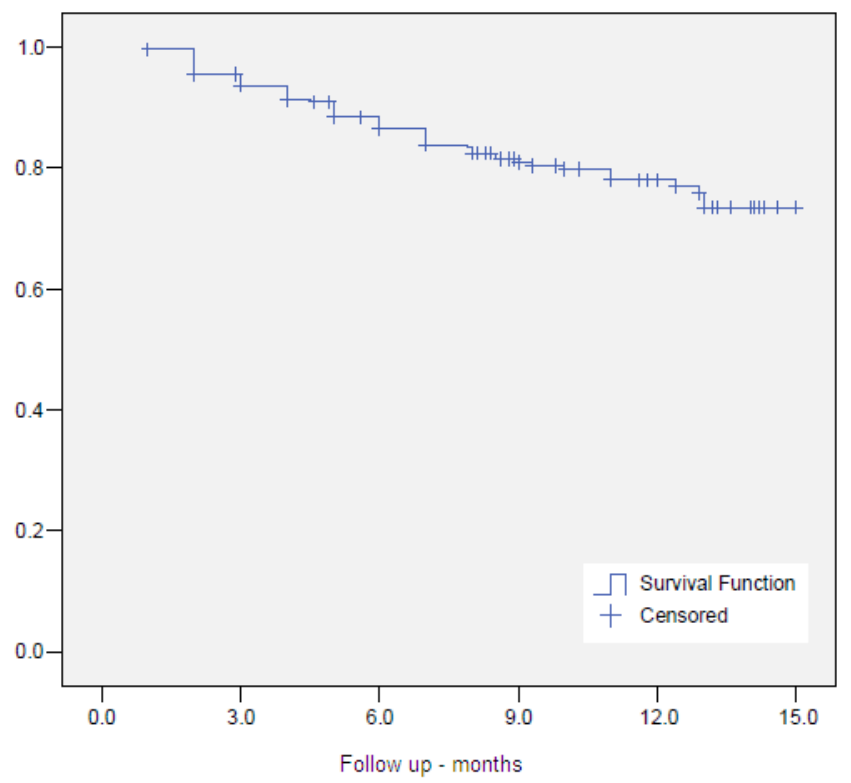

Figure 2: Clinical Specific Survival

Low risk group compared to intermediate, (HR 0.70, 95\% CI 0.023-0.213, $\mathrm{p}<0.001)$ and high risk (HR 0.99, 95\% CI 0.052-0.190, $\mathrm{p}<0.001$ ) groups, was a favorable predictive factor for BC. GS $>7$ (HR $3.09,95 \%$ CI 1.473-6.473, $\mathrm{p}=0.003)$ and PSAi $>10$ (HR 6.18, 95\% CI 2.331-16.393, $\mathrm{p}<0.001$ ) were negative predictive factor for $\mathrm{BC}$.

Low risk group was confirmed as the only predictive factor for OS when compared to intermediate (HR 0.28, 95\% CI 0.133-0.608, $\mathrm{p}=0.001$ ) and high (HR 0.38, 95\% CI 0.223-0.665, $\mathrm{p}=0.001$ ) risk groups. 


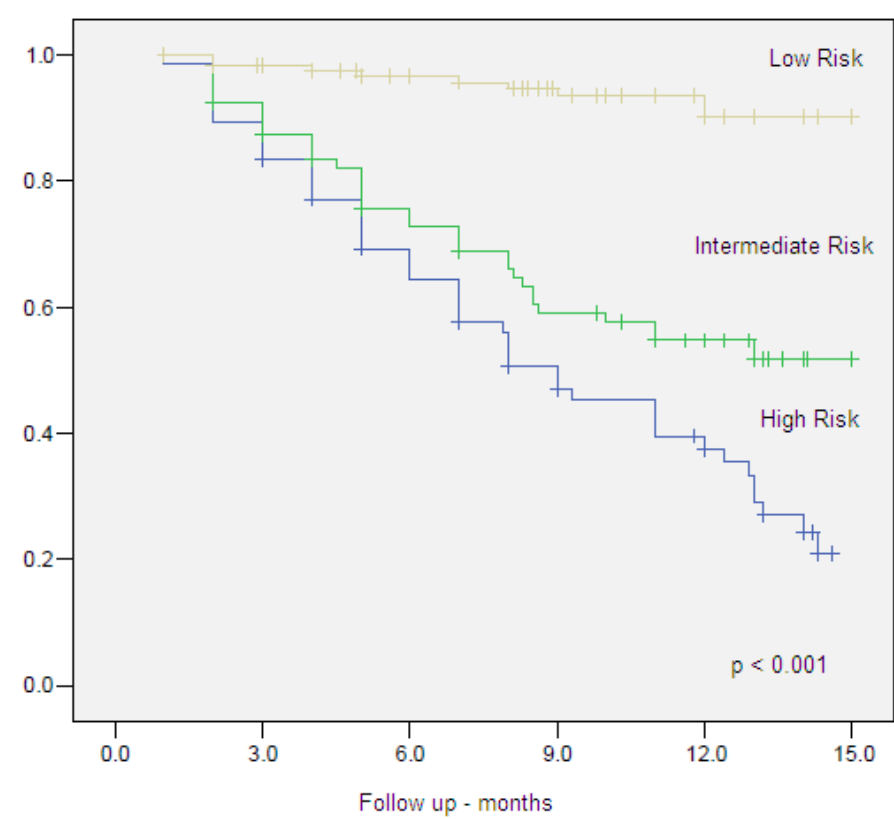

Figure 3: Clinical Specific Survival by Risk Group

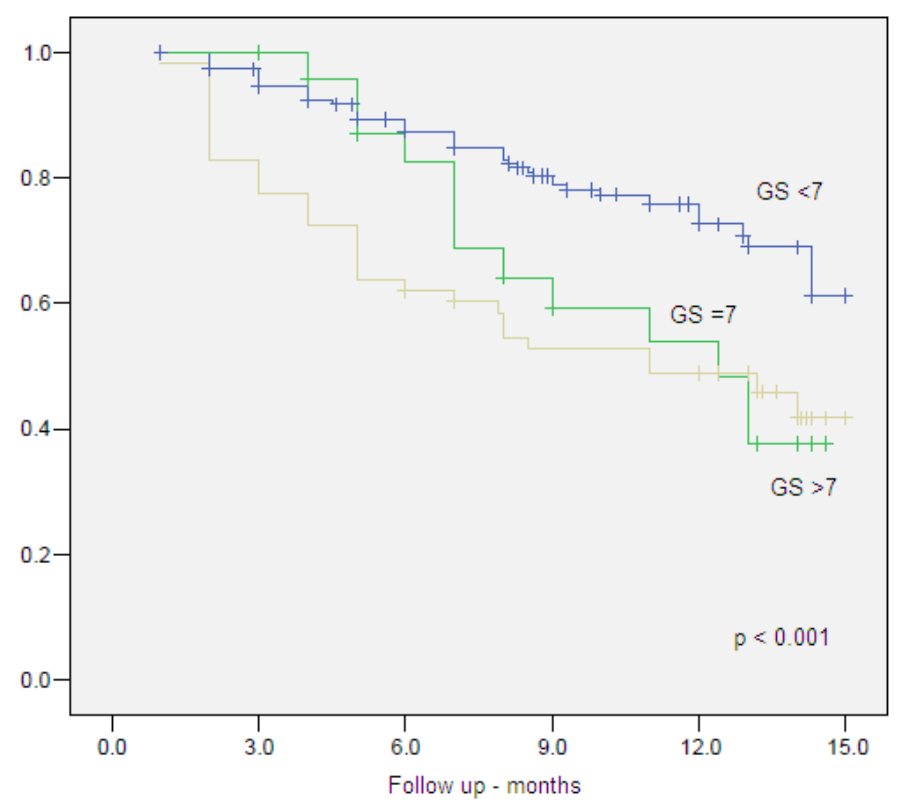

Figure 5: Clinical Specific Survival by Gleason Score

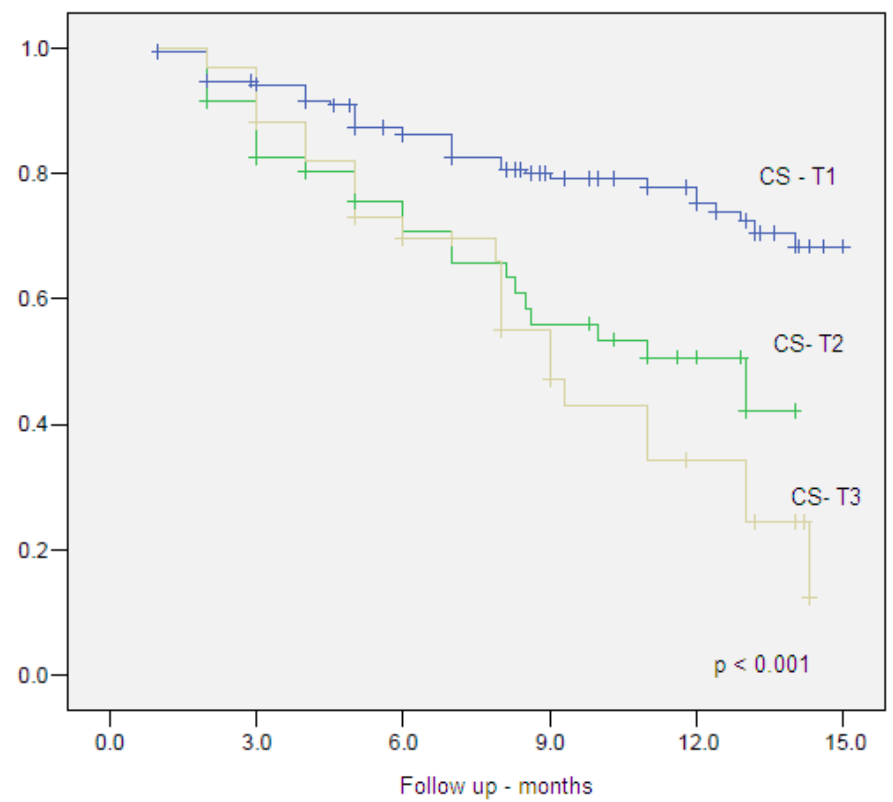

Figure 4: Clinical Specific Survival by Clinical Stage

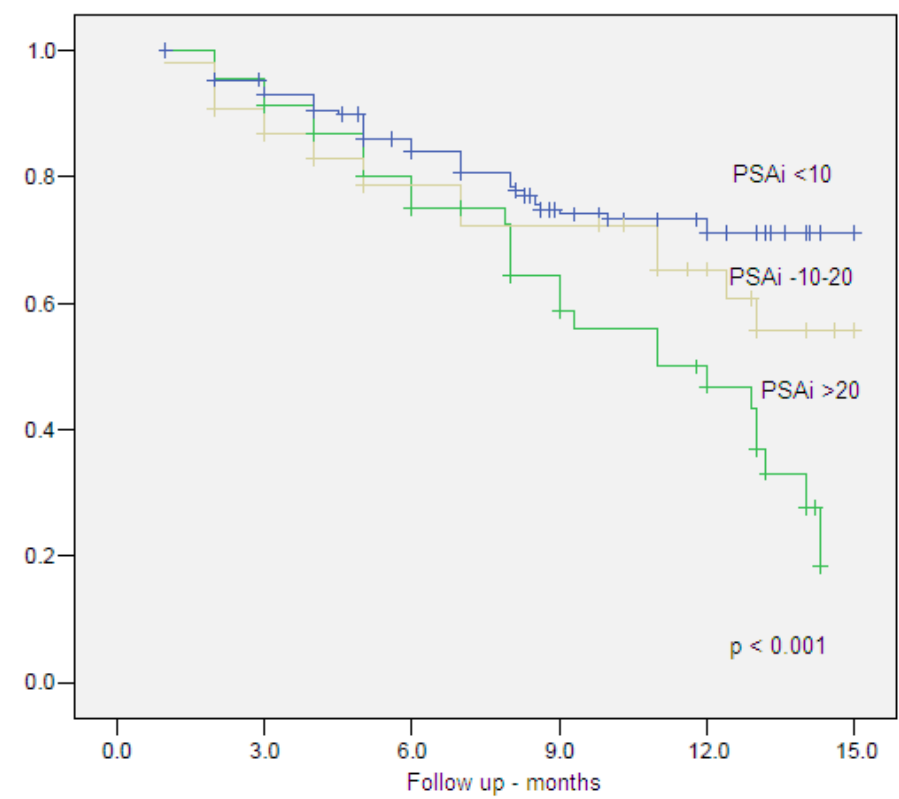

Figure 6: Clinical Specific Survival by Initial PSA 


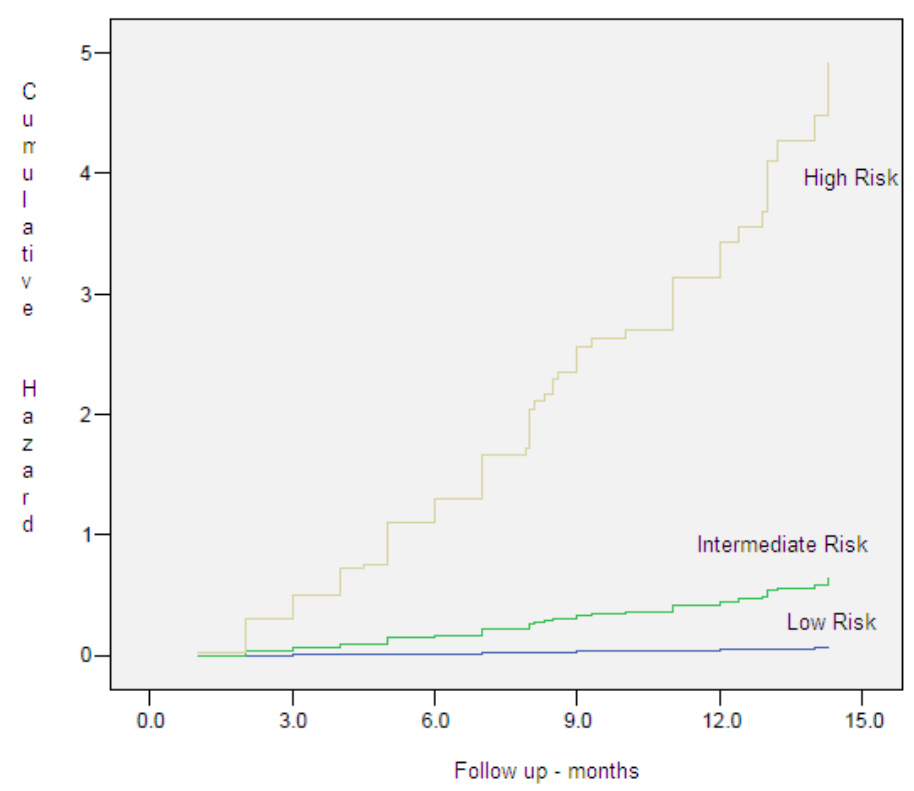

Figure 7: Hazard Plots - Clinical Specific Survival by Risk Group

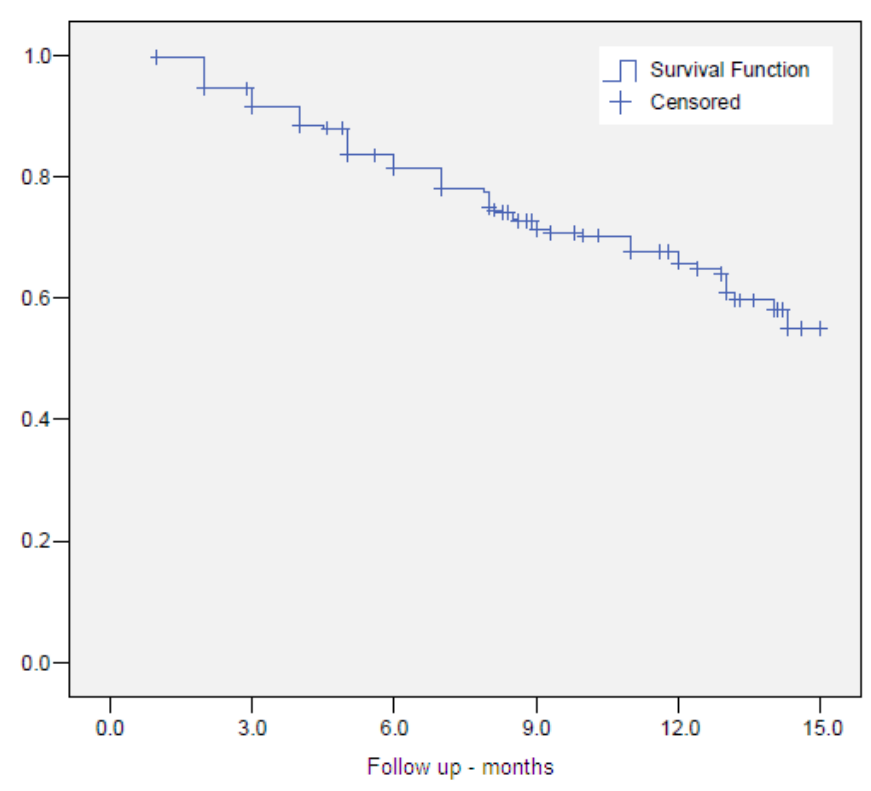

Figure 8: Biochemical Control

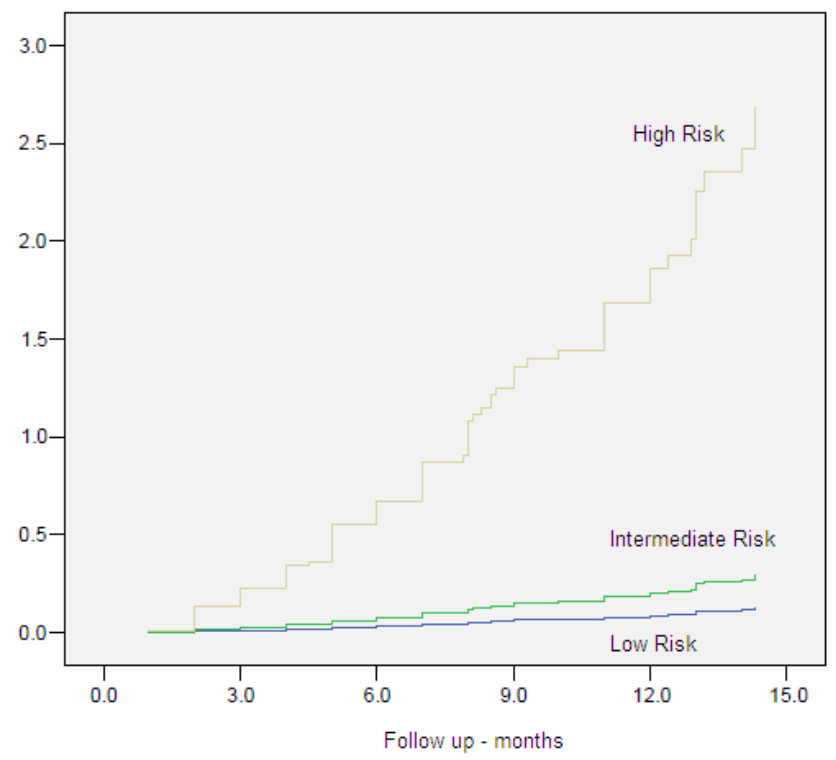

Figure 9: Hazard Plots - Biochemical Control by Risk Group 
Antonio Cassio Assis Pellizzon (2017) Long Term Results of High Dose Rate Brachytherapy and External Beam Radiotherapy for Local and Locally Advanced Prostate Cancer

Table 5: Univariate Analysis

CSS

BC

OS

\begin{tabular}{|c|c|c|c|c|c|c|c|c|c|c|c|}
\hline & & $\mathbf{N}$ & $\mathrm{C}$ & $\%$ & $\mathbf{P}$ & $\mathrm{C}$ & $\%$ & $\mathbf{P}$ & $\mathrm{C}$ & $\%$ & \\
\hline \multirow[t]{3}{*}{ Risk } & Low & 82 & 9 & 11.0 & $<0.001$ & 5 & 6.1 & $<0.001$ & 17 & 6.2 & $<0.001$ \\
\hline & Interm & 100 & 19 & 19.0 & & 5 & 5.0 & & 33 & 12.1 & \\
\hline & High & 91 & 61 & 67.0 & & 24 & 26.4 & & 49 & 17.9 & \\
\hline \multirow[t]{2}{*}{ HDR Plan } & $2 \mathrm{D}$ & 167 & 74 & 44.3 & $<0.001$ & 30 & 18.0 & $<0.001$ & 56 & 20.5 & 0.549 \\
\hline & $3 \mathrm{D}$ & 106 & 15 & 14.2 & & 4 & 3.8 & & 43 & 15.8 & \\
\hline \multirow[t]{2}{*}{ ADJ } & No & 152 & 27 & 17.8 & $<0.001$ & 13 & 8.6 & 0.017 & 45 & 16.5 & 0.004 \\
\hline & Yes & 121 & 62 & 51.2 & & 21 & 17.4 & & 54 & 19.8 & \\
\hline \multirow[t]{2}{*}{ NAAD } & No & 226 & 72 & 31.9 & 0.315 & 29 & 12.8 & 0.924 & 80 & 29.3 & 0.269 \\
\hline & Yes & 47 & 17 & 36.2 & & 5 & 10.6 & & 19 & 7.0 & \\
\hline \multirow[t]{3}{*}{ PSAi (ng/mL) } & $<10$ & 173 & 43 & 24.9 & 0.001 & 15 & 8.7 & $<0.001$ & 63 & 23.1 & 0.514 \\
\hline & $10-20$ & 54 & 19 & 35.2 & & 3 & 5.6 & & 23 & 8.4 & \\
\hline & $>20$ & 46 & 27 & 58.7 & & 16 & 34.8 & & 13 & 4.8 & \\
\hline \multirow[t]{3}{*}{ GS } & $<7$ & 190 & 45 & 23.7 & $<0.001$ & 19 & 10.0 & 0.107 & 67 & 24.5 & 0.728 \\
\hline & $=7$ & 58 & 31 & 53.4 & & 12 & 20.7 & & 21 & 7.7 & \\
\hline & $>7$ & 25 & 13 & 52.0 & & 3 & 12.0 & & 11 & 4.0 & \\
\hline \multirow[t]{3}{*}{$\mathrm{CS}$} & $<\mathrm{T} 2 \mathrm{~b}$ & 192 & 45 & 23.4 & $<0.001$ & 13 & 6.8 & $<0.001$ & 69 & 25.3 & 0.588 \\
\hline & T2b-c & 47 & 22 & 46.8 & & 9 & 19.1 & & 19 & 7.0 & \\
\hline & $>\mathrm{T} 2 \mathrm{c}$ & 34 & 22 & 64.7 & & 12 & 35.3 & & 11 & 4.0 & \\
\hline \multirow[t]{2}{*}{ Age (years) } & $<65 y$ & 151 & 62 & 41.1 & 0.001 & 21 & 13.9 & 0.264 & 61 & 22.3 & 0.062 \\
\hline & $>65 y$ & 122 & 27 & 22.1 & & 13 & 10.7 & & 38 & 13.9 & \\
\hline \multirow[t]{2}{*}{ P vol. } & $<35 \mathrm{cc}$ & 121 & 40 & 33.1 & 0.775 & 18 & 14.9 & 0.236 & 44 & 16.1 & 0.910 \\
\hline & $>35 \mathrm{cc}$ & 152 & 49 & 32.2 & & 16 & 10.5 & & 55 & 20.1 & \\
\hline \multirow[t]{4}{*}{ EBRT } & $<50 \mathrm{~Gy}$ & 149 & 70 & 47.0 & $<0.001$ & 27 & 18.1 & 0.002 & 55 & 20.1 & 0.397 \\
\hline & $>50 \mathrm{~Gy}$ & 124 & 19 & 15.3 & & 7 & 5.6 & & 44 & 16.1 & \\
\hline & Local & 209 & 54 & 25.8 & $<0.001$ & 22 & 10.5 & 0.055 & 66 & 24.2 & 0.004 \\
\hline & Pelvic & 64 & 35 & 54.7 & & 12 & 18.8 & & 33 & 12.1 & \\
\hline \multirow[t]{2}{*}{ HDR (Gy) } & $<16 \mathrm{~Gy}$ & 133 & 17 & 12.8 & $<0.001$ & 5 & 3.8 & $<0.001$ & 46 & 16.8 & 0.306 \\
\hline & $>16 \mathrm{~Gy}$ & 140 & 72 & 51.4 & & 29 & 20.7 & & 53 & 19.4 & \\
\hline \multirow[t]{2}{*}{ Interval } & $<18 \mathrm{~m}$ & 173 & 57 & 12.1 & 0.882 & 21 & 32.9 & 0.812 & 60 & 22.0 & 0.636 \\
\hline & $>18 \mathrm{~m}$ & 100 & 32 & 13.0 & & 13 & 32.0 & & 39 & 14.3 & \\
\hline \multirow[t]{2}{*}{ Comorb } & No & 146 & 45 & 30.8 & 0.117 & 18 & 12.3 & 0.467 & 47 & 17.2 & 0.040 \\
\hline & Yes & 127 & 44 & 34.6 & & 16 & 12.6 & & 52 & 19.0 & \\
\hline Total & & 273 & 89 & 36.2 & & 34 & 12.5 & & 99 & 36.3 & \\
\hline
\end{tabular}

Legend: ADJ (adjuvant hormonal therapy), BC (biochemical control), C (Censored), Comorb (Comorbidites), CSS (Clinical Specific Survival), EBRT (External beam radiotherapy), Interval $\mathrm{m}$ (Interval in months), NAAD (neoadjuvant hormonal therapy), OS (overall survival), P vol. (Prostate volume cc), Plan 2D/3D (two or three dimensional planning) 
Table 6: Cox regression for CSS

\begin{tabular}{|c|c|c|c|c|c|c|c|c|}
\hline & B & SE & Wald & df & Sig. & $\operatorname{Exp}(B)$ & \multicolumn{2}{|c|}{$95.0 \%$ CI for $\operatorname{Exp}(B)$} \\
\hline & & & & & & & Lower & Upper \\
\hline LR & & & 44.294 & 2 & .000 & & & \\
\hline IR & -3.641 & .761 & 22.910 & 1 & .000 & .026 & .006 & .116 \\
\hline HR & -2.351 & .418 & 31.579 & 1 & .000 & .095 & .042 & .216 \\
\hline EBRT Pelvic $x$ Local & -.110 & .312 & .124 & 1 & .724 & .896 & .486 & 1.651 \\
\hline $2 D \times 3 D$ & -1.861 & 1.134 & 2.693 & 1 & .101 & .156 & .017 & 1.436 \\
\hline ADT & -.931 & .403 & 5.336 & 1 & .021 & .394 & .179 & .868 \\
\hline PSAi $<10$ & & & 10.858 & 2 & .004 & & & \\
\hline PSAi $(>10<20)$ & 1.925 & .661 & 8.481 & 1 & .004 & 6.856 & 1.877 & 25.048 \\
\hline PSAi (>20) & 1.824 & .573 & 10.133 & 1 & .001 & 6.195 & 2.015 & 19.041 \\
\hline GS $<7$ & & & 7.328 & 2 & .026 & & & \\
\hline GS $=7$ & .335 & .709 & .223 & 1 & .636 & 1.398 & .349 & 5.608 \\
\hline GS $>7$ & 1.176 & .475 & 6.145 & 1 & .013 & 3.243 & 1.279 & 8.220 \\
\hline $\mathrm{CS}<\mathrm{T} 2 \mathrm{~b}$ & & & 3.350 & 2 & .187 & & & \\
\hline $\mathrm{CS}$ T2b/T2c & -.662 & .551 & 1.443 & 1 & .230 & .516 & .175 & 1.519 \\
\hline $\mathrm{CS}>\mathrm{T} 2 \mathrm{c}$ & .464 & .818 & .321 & 1 & .571 & 1.590 & .320 & 7.895 \\
\hline Age $<65 y$ & 1.053 & .418 & 6.352 & 1 & .012 & 2.867 & 1.264 & 6.504 \\
\hline EBRT < 50Gy & 2.674 & 1.044 & 6.562 & 1 & .010 & 14.498 & 1.874 & 112.164 \\
\hline HDR dose <20Gy & 1.763 & 1.023 & 2.973 & 1 & .085 & 5.830 & .786 & 43.261 \\
\hline Interval EBRT to HDR & -.187 & .303 & .381 & 1 & .537 & .829 & .458 & 1.502 \\
\hline
\end{tabular}

Legend: 2D (two dimensional plan), 3D (tridimensional plan), ADT (Androgen deprivation therapy), BC (biochemical control), Comorb (Comorbidites), CS (Clinical stage), CSS (Clinical Specific Survival), EBRT (External beam radiotherapy), GS (Gleason score), IR (Intermediate risk group), HDR (High-dose-rate brachytherapy), HR (High risk group), LR (Low risk group), NAAD (neoadjuvant hormonal therapy), OS (overall survival), P vol. (Prostate volume cc).

Table 7: Cox regression for $\mathrm{BC}$

\begin{tabular}{|c|c|c|c|c|c|c|c|c|}
\hline & B & SE & Wald & df & Sig. & $\operatorname{Exp}(B)$ & \multicolumn{2}{|c|}{$95.0 \%$ CI for $\operatorname{Exp}(B)$} \\
\hline & & & & & & & Lower & Upper \\
\hline LR & & & 62.053 & 2 & .000 & & & \\
\hline IR & -2.660 & .568 & 21.948 & 1 & .000 & .070 & .023 & .213 \\
\hline HR & -2.309 & .330 & 48.920 & 1 & .000 & .099 & .052 & .190 \\
\hline $2 D \times 3 D$ & -.789 & .656 & 1.448 & 1 & .229 & .454 & .126 & 1.642 \\
\hline ADT & -.455 & .307 & 2.192 & 1 & .139 & .634 & .347 & 1.159 \\
\hline PSAi $<10$ & & & 13.634 & 2 & .001 & & & \\
\hline PSAi $(>10<20)$ & 1.822 & .498 & 13.403 & 1 & .000 & 6.182 & 2.331 & 16.393 \\
\hline PSAi $>20$ & 1.346 & .462 & 8.494 & 1 & .004 & 3.843 & 1.554 & 9.502 \\
\hline GS $<7$ & & & 8.944 & 2 & .011 & & & \\
\hline GS $=7$ & .957 & .465 & 4.240 & 1 & .039 & 2.603 & 1.047 & 6.469 \\
\hline GS $>7$ & 1.127 & .378 & 8.915 & 1 & .003 & 3.088 & 1.473 & 6.473 \\
\hline $\mathrm{CS}<\mathrm{T} 2 \mathrm{~b}$ & & & 2.825 & 2 & .244 & & & \\
\hline $\operatorname{CST} \mathbf{T} b / T 2 c$ & -.647 & .410 & 2.493 & 1 & .114 & .523 & .234 & 1.169 \\
\hline $\mathrm{CS}>\mathrm{T} 2 \mathrm{c}$ & -.306 & .580 & .278 & 1 & .598 & .737 & .236 & 2.294 \\
\hline EBRT 50Gy & 1.411 & .550 & 6.590 & 1 & .010 & 4.101 & 1.396 & 12.044 \\
\hline HDR dose < 20Gy & -.470 & .680 & .477 & 1 & .490 & .625 & .165 & 2.370 \\
\hline Interval EBRT to HDR & -.064 & .230 & .078 & 1 & .780 & .938 & .597 & 1.473 \\
\hline
\end{tabular}

Legend: 2D (two dimensional plan), 3D (tridimensional plan), ADT (Androgen deprivation therapy), BC (biochemical control, Comorb (Comorbidites), CS (Clinical stage), CSS (Clinical Specific Survival), EBRT (External beam radiotherapy), GS (Gleason score), IR (Intermediate risk group), HDR (High-dose-rate brachytherapy), HR (High risk group), LR (Low risk group), NAAD (neoadjuvant hormonal therapy), OS (overall survival), P vol. (Prostate volume). 
Table 8: Cox regression for OS

\begin{tabular}{|l|l|l|l|l|l|l|l|l|}
\hline & B & SE & Wald & df & Sig. & $\operatorname{Exp(B)}$ & \multicolumn{2}{|c|}{$\begin{array}{c}\text { E5.0\% CI for } \\
\operatorname{Exp}(B)\end{array}$} \\
\hline & & & & & & & Lower & Upper \\
\hline ADJ & .149 & 0.3 & 0.3 & 1 & .580 & 1.161 & .684 & 1.970 \\
\hline Age $<\mathbf{6 5 y}$ & .223 & 0.2 & 1.0 & 1 & .322 & 1.250 & .804 & 1.942 \\
\hline $\begin{array}{l}\text { Comor- } \\
\text { bidity }\end{array}$ & -.310 & 0.2 & 2.1 & 1 & .149 & .734 & .482 & 1.117 \\
\hline $\begin{array}{l}\text { EBRT Pel- } \\
\text { vic x Local }\end{array}$ & -.089 & 0.2 & 0.1 & 1 & .722 & .915 & .560 & 1.494 \\
\hline LR & & & 14.3 & 2 & .001 & & & \\
\hline IR & -1.259 & 0.4 & 10.5 & 1 & .001 & .284 & .133 & .608 \\
\hline HR & -.954 & 0.3 & 11.7 & 1 & .001 & .385 & .223 & .665 \\
\hline
\end{tabular}

Legend: ADJ (adjuvant hormonal therapy, EBRT (External beam radiotherapy), IR (Intermediate risk group), HDR, HR (High risk group), LR (Low risk group), OS (overall survival)

Table 9: Results of biochemical control by risk groups in series of HDR plus EBRT with more than 5-year follow up

\begin{tabular}{|c|c|c|c|c|c|c|}
\hline Reference & $\mathbf{n}$ & $\begin{array}{c}\text { Median FU } \\
\text { (months) }\end{array}$ & \multicolumn{3}{|c|}{$\begin{array}{c}\text { Biochemical control by risk } \\
\text { groups (\%) } \\
\text { Low Intermediate High }\end{array}$} & $\begin{array}{c}\text { Dose in Gy } \\
\text { (HDR(n.fx)/ } \\
\text { EBRT) }\end{array}$ \\
\hline 13 & 344 & 61 & & 84 & 74 & $19.5(3) / 46$ \\
\hline 14 & 121 & 63 & & 91 & & $10(1) / 50$ \\
\hline 15 & 313 & 68 & 100 & 88 & 79 & $23(2) / 46$ \\
\hline 16 & 229 & 61 & 95 & 90 & 57 & $21(3) / 50.4$ \\
\hline 17 & 64 & 105 & & 84 & 80 & $18(3) / 45$ \\
\hline 18 & 90 & 95 & & & 80 & $16.5(3) / 45$ \\
\hline 19 & 264 & 75 & 97 & & & $18(3) / 45$ \\
\hline 20 & 100 & 62 & & 84 & 82 & $10(1) / 60$ \\
\hline 21 & 64 & 61 & & 100 & 91 & $21(3) / 50$ \\
\hline 22 & 196 & 66 & & 86 & & $18(3) / 46$ \\
\hline 23 & 131 & 63 & & 87 & 71 & $30(4) / 45$ \\
\hline
\end{tabular}

\section{Discussion}

Conventional EBRT to treat PCa is securely limited to doses of 64-70 Gy in 1.8-2.0 Gy fractions. These levels of doses are determined by the risk of long-term toxic effects to the bladder and rectum. The clinical and biochemical relapse rates associated with these dose levels are around 33\% within 5 years. Peeters et al ${ }^{12}$ published the results of a randomized trial comparing total doses of 68 Gy and 78 Gy using EBRT alone. The 5-year OS in the higher dose arm of that study was 83\% using ASTRO definition and the BC was significantly better in the 78-Gy arm compared to the 68-Gy arm, with an adjusted hazard ratio of $0.74(\mathrm{p}=0.02)$. Other published reports using dose-escalated photon beam EBRT alone also point in the same direction with respect to their long-term BC results [13-14].

HDR can escalate the dose given to the prostate by the combination with EBRT, and further more, in locally advanced disease has also the possibility of including the seminal vesicles when they needed to be encompassed. HDR has also a potential biological advantage through the delivery of high doses per fraction [10]. It is important to note that the comparisons between series published are difficult due differences in the techniques and planning for both, EBRT and HDR.
The combination of values of PSAi, GS and CS to identify a more or less aggressive disease has being extensively discussed in the literature, and was confirmed by this study. The most frequent challenge is to identify, for example the indication of prostate versus pelvic EBRT, varying risk categories, absence or use of NAAD, ADT and their length. Despite this, the combination of HDR and EBRT provides an optimal modulation of dose delivery. Results of this combination, in terms of $\mathrm{BC}$ and with more than 5 years of $\mathrm{FU}$, range from $57 \%$ to $100 \%$ according to the risk group for biochemical failure (Table 9).

The search for factors predicting BC and CSS is important on defining what patients should be treated more aggressively. We, as other authors [26-28] observed that PSAi $<10 \mathrm{ng} / \mathrm{ml}$ was confirmed as a favorable predictive factor related to $\mathrm{BC}$ and CSS.

Age $<65$ years was found to be an adverse prognostic factor in our analysis for CSS, with a marginally statically significance impact on OS, not confirmed on multivariate analysis. Smolska-Ciszewska et al, conversely to our results, noted that younger age at time of treatment impacted only on OS, not explaining if there was any association between treatment and side effects or worsening of associated comorbidities. As in our analysis, they found that low risk group and association of HDR to EBRT correlated with improved BC [29].

As in our results, Kamrava et al found that T stage, GS, and use of ADT were significantly associated with CSS on univariate analysis, but on multivariate analysis only GS and use of ADT were significantly associated CSS [30]

It is expected that the grouping of the patients in risk groups for biochemical failure based on PSAi, GS and CS, to aggregate patients with adverse features in the intermediate and high risk, leading to worse CSS and BC for the two last one, what was confirmed in our analysis. This was also observed by Morris et al [31].

In our series, as in others, patients who received adjuvant ADT had significantly higher risk features suggesting patient selection bias for CSS in this group of patients, instead of a negative interaction between HDR and EBRT [31-32].

Mature data in the literature evaluated the 10-year outcomes of intermediate- and high-risk patients noting a clear dose response by increasing the dose escalation through HDR doses [27]. More recently the use of Intensity modulated radiation therapy combined to HDR has being investigated. Chen $\mathrm{et} \mathrm{al}$. published the results of 148 patients treated with HDR - 22 Gy in 4 fractions followed IMRT up to 50.4Gy. All patients with Gleason score of 8 or higher had ADT for 1 year. They noted a 4 -year actuarial CSS of $96.8 \%$ and of $100 \%, 100 \%$ and $94 \%$ for low, intermediate and high risk, respectively [32].

The results of the first randomized prospective trial, which has addressed dose escalation using an HDR and EBRT is a trial with a relatively slow accrual rate. There are some critics that must be addressed as the changes in EBRT technique during the time of the study, and, by current standards, the control arm is a relatively lowdose treatment. Despite that, the study reported the results of 218 patients treated between 1997 and 2005. There were 108 patients assigned to EBRT alone and 110 patients treated by EBRT followed by HDR. They noted that CSS was significantly higher in patients treated with combined modality $(p=0.04)$. In multivariate analysis 
the category treatment modality and ADT were significant covariates for BC, but with no differences in OS, as observed in our study. After a median follow-up time of 10.5 year follow-up, an $18 \%$ increase in CSS was obtained relative to EBRT alone, reflecting a $31 \%$ reduction in the risk of recurrence $(p=0.01)$ and no evidence of an increase in long-term severe morbidity [9].

Surgically induced gland deformation is inevitable during brachytherapy procedures. We observed that the use more of advanced methods of images (3D plan based on CT, MR or TRUS) images to identify the target, organs at risk and needles is important and have already been reported to impact on BC and CSS [33]. In our analysis Multivariate Cox regression analysis confirmed EBRT dose >50 Gy as predictor for CSS and BC, but with no impact on OS. This may be explained by the relative low dose per fraction schedule used in both groups. Of importance is to note that the dose given by HDR was relative constant thorough the risk groups, leading to higher biological effective dose to intermediate and high risk patients, and even though, these patients had a worse outcome, showing that there is space for further studies of dose escalation or treatment combination. After 2005 with the introduction of real time TRUS image acquisition and planning we have changed our protocol and moved forward for a more intense dose escalation, increasing dose and reducing the number of fractions.

Other information of this study is that presence of NAAD had no impact on DSS, BC or OS in any risk group, showing that this treatment strategy should be reserved for downsizing the prostate prior to treatment, in special for low risk patients.

In conclusion, this report demonstrates that HDR combined with EBRT is an important and effective method in achieving dose escalation in the radical radiotherapy of PCa. This combination has also the advantage of treatment time reduction and in increasing in the capability of work load of the linear accelerators, especially in developing countries, where waiting lists and lack of radiation oncology facilities are a reality. The present data represents a unique uni-institutional study at long FU for the given technique, and a comparison with the current literature confirms the excellent results achieved with this treatment modality. The satisfactory BC, CSS and OS are probably result of improved LC achieved with dose escalation, showing that HDR is an optimal alternative method of local dose escalation when combined to EBRT.

\section{References}

1. Quinn MJ, d'Onofrio A, Møller B, Black R, Martinez-Garcia C, et al. (2003) Cancer mortality trends in the EU and acceding countries up to 2015. Ann Oncol 14: 11481152. [crossref]

2. http: //www.inca.gov.br/estimativa/2014/sintese-de-resultados- comentarios.asp

3. Novara G, Ficarra V, Mocellin S, et al. (2012) Systematic review and meta-analysis of studies reporting oncologic outcome after robot-assisted radical prostatectomy. Eur Urol 62: 382.

4. Al-Mamgani A, van Putten WL, Heemsbergen WD, van Leenders GJ, Slot A, et al. (2008) Update of Dutch multicenter dose-escalation trial of radiotherapy for localized prostate cancer. Int J Radiat Oncol Biol Phys 72: 980-988. [crossref]

5. Dearnaley DP, Sydes MR, Graham JD, et al. (2007) Escalated-dose versus standarddose conformal radiotherapy in prostate cancer: first results from the MRC RT01 randomized controlled trial. Lancet Oncol 8: 475.

6. Kuban DA, Tucker SL, Dong L, Starkschall G, Huang EH, et al. (2008) Long-term results of the M. D. Anderson randomized dose-escalation trial for prostate cancer. Int J Radiat Oncol Biol Phys 70: 67-74. [crossref]
7. Zietman AL, DeSilvio ML, Slater JD, et al. (2005) Comparison of conventional-dose vs high-dose conformal radiation therapy in clinically localized adenocarcinoma of the prostate: a randomized controlled trial. JAMA 294: 1233

8. Pellizzon AC, Salvajoli JV, Maia MA, et al. (2004) Late urinary morbidity with high dose prostate brachytherapy as a boost to conventional external beam radiation therapy for local and locally advanced prostate cancer. J Urol 171: 1105.

9. Hoskin PJ, Rojas AM, Bownes PJ, et al. (2012) Randomized trial of external beam radiotherapy alone or combined with high-dose-rate brachytherapy boost for localized prostate cancer. Radiother Oncol 103: 217.

10. Pellizzon AC, Nadalin W, Salvajoli JV, Fogaroli RC, et al. (2003) Results of high dose rate afterloading brachytherapy boost to conventional external beam radiation therapy for initial and locally advanced prostate cancer. Radiother Oncol 66: 167.

11. Roach M 3rd, Hanks G, Thames H Jr, et al. (2006) Defining biochemical failure following radiotherapy with or without hormonal therapy in men with clinically localized prostate cancer: recommendations of the RTOG-ASTRO Phoenix Consensus Conference. Int J Radiat Oncol Biol Phys 65: 965.

12. Peeters ST, Heemsbergen WD, Koper PC, et al. (2006) Dose-response in radiotherapy for localized prostate cancer: results of the Dutch multicenter randomized phase III trial comparing 68 Gy of radiotherapy with 78 Gy. J Clin Oncol 24.

13. Pollack A, Zagars GK, Starkschall G, Antolak JA, Lee JJ, et al. (2002) Prostate cancer radiation dose response: results of the M. D. Anderson phase III randomized trial. Int J Radiat Oncol Biol Phys 53: 1097-1105. [crossref]

14. Dearnaley DP, Sydes MR, Graham JD, et al. (2007) Escalated dose versus standarddose conformal radiotherapy in prostate cancer: first results from the MRC RT01 randomized controlled trial. Lancet Oncol 8: 475.

15. Khor R, Duchesne G, Tai KH, et al. (2013) Direct 2-arm comparison shows benefit of high-dose-rate brachytherapy boost vs external beam radiation therapy alone for prostate cancer. Int J Radiat Oncol Biol Phys 85: 679.

16. Cury FL, Duclos M, Aprikian A, et al. (2012) Single-fraction high-dose-rate brachytherapy and hypofractionated external beam radiation therapy in the treatment of intermediate-risk prostate cancer - long term results. Int J Radiat Oncol Biol Phys 82: 1417.

17. Prada PJ, González H, Fernández J, Jiménez I, Iglesias A, et al. (2012) Biochemical outcome after high-dose-rate intensity modulated brachytherapy with external beam radiotherapy: 12 years of experience. BJU Int 109: 1787-1793. [crossref]

18. Kotecha R, Yamada Y, Pei X, Kollmeier MA, Cox B, et al. (2013) Clinical outcomes of high-dose-rate brachytherapy and external beam radiotherapy in the management of clinically localized prostate cancer. Brachytherapy 12: 44-49. [crossref]

19. Kaprealian T, Weinberg V, Speight JL, et al. (2012) High-dose-rate brachytherapy boost for prostate cancer: comparison of two different fractionation schemes. Int $J$ Radiat Oncol Biol Phys 82: 222.

20. Savdie R, Symons J, Spernat D, Yuen C, Pe Benito RA, et al. (2012) High-dose rate brachytherapy compared with open radical prostatectomy for the treatment of high-risk prostate cancer: 10 year biochemical freedom from relapse. BJU Int 110 Suppl 4: 71-76. [crossref]

21. Aluwini S, van Rooij PH, Kirkels WJ, Jansen PP, Praag JO, et al. (2012) Highdose-rate brachytherapy and external-beam radiotherapy for hormone-naïve lowand intermediate-risk prostate cancer: a 7-year experience. Int J Radiat Oncol Biol Phys 83: 1480-1485. [crossref]

22. Agoston P, Major T, Fröhlich G, et al. (2011) Moderate dose escalation with singlefraction high-dose-rate brachytherapy boost for clinically localized intermediateand high-risk prostate cancer: 5-year outcome of the first 100 consecutively treated patients. Brachytherapy 10: 376

23. Ghadjar P, Keller T, Rentsch CA, Isaak B, Behrensmeier F, et al. (2009) Toxicity and early treatment outcomes in low- and intermediate-risk prostate cancer managed by high-dose-rate brachytherapy as a monotherapy. Brachytherapy 8: 45-51. [crossref]

24. Zwahlen DR, Andrianopoulos N, Matheson B, Duchesne GM, Millar JL (2010) High-dose-rate brachytherapy in combination with conformal external beam radiotherapy in the treatment of prostate cancer. Brachytherapy 9: 27-35. [crossref]

25. Viani GA, Pellizzon AC, Guimarães FS, Jacinto AA, dos Santos Novaes PE, et al. (2009) High dose rate and external beam radiotherapy in locally advanced prostate cancer. Am J Clin Oncol 32: 187-190. [crossref]

26. Aoki M, Miki K, Kido M, et al. (2014) Analysis of prognostic factors in localized high-risk prostate cancer patients treated with HDR brachytherapy, hypofractionated 3D-CRT and neoadjuvant/adjuvant androgen deprivation therapy (trimodality therapy). J Radiat Res 55: 527.

27. Martinez AA, Gonzalez J, Ye H, et al. (2011) Dose escalation improves cancerrelated events at 10 years for intermediate- and high-risk prostate cancer patients treated with hypofractionated high-dose-rate boost and external beam radiotherapy. Int J Radiat Oncol Biol Phys 79: 363.

28. Shigehara K, Mizokami A, Komatsu K, Koshida K, Namiki M (2006) Four year clinical statistics of iridium-192 high dose rate brachytherapy. Int J Urol 13: 116121. [crossref]

29. Smolska-Ciszewska B, Miszczyk L, Bialas B, et al. (2015) The effectiveness and side effects of conformal external beam radiotherapy combined with high-dose-rate brachytherapy boost compared to conformal external beam radiotherapy alone in patients with prostate cancer. Radiat Oncol 10: 366. 
30. Kamrava M, Rwigema JC, Chung M, Banerjee R, Wang J, et al. (2013) Predictors of distant metastasis after combined HDR brachytherapy and external beam radiation for prostate cancer. J Contemp Brachytherapy 5: 127-133. [crossref]

31. Morris LM, Izard MA, Wan WY (2015) Does prostate-specific antigen nadir predict longer-term outcome es of prostate cancer after neoadjuvant and adjuvant androgen
deprivation therapy in conjunction with brachytherapy? Brachytherapy 14: 322 . outcome

32. Chen WC, Tokita KM, Ravera J, Fu P, Jiang Y, et al. (2013) Four-year outcomes of hypofractionated high-dose-rate prostate brachytherapy and external beam radiotherapy. Brachytherapy 12: 422-427. [crossref]

33. Pellizzon AC, Salvajoli J, Novaes P, et al. (2008) The relationship between the biochemical control outcomes and the quality of planning of high-dose rate brachytherapy as a boost to external beam radiotherapy for locally and locally advanced prostate cancer using the RTOG-ASTRO Phoenix definition. Int J Med Sci 5: 113.

\section{Abbreviations and Acronyms}

ADT: Adjuvant Androgen Deprivation

AJCC: American Joint Committee on Cancer

BC: Biochemical Control

CSS: Clinical Specific Survival

EBRT: External Beam Radiotherapy

FU: Fallow Up

GS: Gleason Score

HDR: High-Dose-Rate Brachytherapy

LR: Low Risk Group

NAAD: Neoadjuvant Hormonal Therapy

OS: Overall Survival

PSAi: Initial Prostate-Specific Antigen

TRUS: Trans Rectal Ultrasound

\section{Citation:}

Antonio Cassio Assis Pellizzon, Deivid Augusto da Silva, Ricardo Cesar Fogaroli, Maria Leticia Gobo Silva, et al. (2017) Long Term Results of High Dose Rate Brachytherapy and External Beam Radiotherapy for Local and Locally Advanced Prostate Cancer. Cancer Stud Ther J Volume 2(1): 1-10 